\title{
Roux-en-Y longitudinal Pancreatico Jejunostomy for Pancreatic Calculi in Children
}

\author{
AKM Zahid Hossain ${ }^{1}$, Gazi Zahirul Hasan², Shafiqul Hoque ${ }^{3}$ \\ ${ }^{1}$ Assistant Professor, Department of Paediatric Surgery, BSMMU, ${ }^{2}$ Assistant Professor, Department of Paediatric Surgery, BSMMU, \\ ${ }^{3}$ Chairman, Department of Paediatric Surgery, BSMMU, Dhaka
}

\begin{abstract}
:
Background: Pancreatic calculi is distinctly uncommon in children and usually present wit recurrent abdominal pain. Objective: To assess the effectiveness of longitudinal pancreatico-jejunostomy in relieving intractable abdominal pain in children with pancreatic calculi. Methods: this prospective study was conducted from 2003-2009 at Paediatric Surgery Department of BSMMU. Ductal decompression by longitudinal pancreatic jejunostomy (LPJ) was done in 14 children (10 male, 4 female) suffering from intractable abdominal pain due to pancreatic calculi. The operative outcomes were divided in to satisfactory and unsatisfactory according to whether the patients were completely or almost completely relieved of pain or continued to be troubled by pain. The main outcome measures were pain relief, postoperative morbidity and mortality. Results: There was no mortality and no significant postoperative morbidity. 14 patients were followed up till 2009. The mean follow-up period were 22 (range 8-72) months. Operative results was satisfactory (no pain) in 12 patients and unsatisfactory (moderate pain) in 2 patients. Complete pain relief was seen 12 patients. Conclusion: Longitudianal pancreatico-jejunostomy is a good operative procedure to relieve intractable abdominal pain in patients with pancreatic calculi.
\end{abstract}

Key words: Pancreatic calculi, Pancreatico-jejunostomy, abdominal pain.

[BSMMU J 2010; 3(2): 72-75]

\section{Introduction:}

Pancreatic calcification is categorized into pancreatic duct calculi (real stone) and pancreatic parenchymal calcification (false stone). It has extremely low incidence in children. Pancreatic calcification often associated with chronic pancratitis ${ }^{1}$. Surgical treatment of pancreatic calculi directed mainly towards the relief of severe abdominal pain.

Three surgical approaches have been described: decompression or drainage operations, pancreatic resection and denervation procedures, The choice of the procedure is based on the main pancreatic duct (MPD) morphology. Drainage procedure have generally been recommended for children with refractory pain with dilated $\mathrm{MPD}^{2}$. This study was done to evaluate the effectiveness of Roux-en-Y longitudinal pancreatico jejunostomy (LPJ) in controlling intractable abdominal pain due to pancreatic duct calculi in children.

\section{Methods:}

This study conducted between May 2003 to December 2009 in Paediatric Surgery Department, BSMMU, 14

Corresponding author: Dr. A.K.M. Zahid Hossain, Room no. 217, Block-C, Department of Paediatric Surgery, Bangabandhu Sheikh Mujib Medical University, Dhaka, E-mail address: drzahidps@gmail.com children with pancreatolithiasis were included. The median age of the patients in this series was 10 (range 7-15) years. Mean duration of illness was 2.35 (range 1.5 -4.5) years. The inclusion criteria were pancreatic duct stone with severe recurrent abdominal pain with an main pancreatic duct (MPD) diameter of $>5 \mathrm{~mm}$.

Pain severity was assessed both pre-operatively and postoperatively was categorized as none to mild if patients had no pain or pain which did not interfere with everyday life and required nothing stronger than traditional simple analgesics.

Moderate pain is intermittent or constant, severe enough to require some oral analgesics frequently. Severe pain required hospitalization, warranted administration of more potent narcotics because of its unbearable, intermittent or constant nature.

Though tools such as the visual analogue scale are very useful for pain assessment, we had opted for the relatively simpler means of evaluating pain verbally as it required the patients to passes minimal verbal and language recognition skills only.

Disease related exclusion criteria were pancreatic calculi with small duct diameter of $<5 \mathrm{~mm}$. Patient related exclusion 
criteria were gross cardiovascular abnormality or coexisting malignancy of pancrease or other organs.

Diagnosis of pancreatic calculi was made from suggestive history, clinical examinations and routine diagnostic workup like x-ray abdomen, ultrasonography, MRCP (Magnatic resonence cholangio-pancreatogram) and CT scan of the abdomen.

The x-ray was useful in detecting stone in 8 out of 10 cases where it was advised. USG was done in all 14 patients and gave positive informations in all. MRCP was performed in 10 and gave positive information. CT scan was done in 4 patients and gave positive informations. The median MPD diameter was 7 (range 5-10)mm. All children underwent ductal decompression by longitudinal pancreatico jejunostomy.

Operative Procedure:

All ductal decompressions of this series were primary procedures. The pancrease was approached via the gastrocolic omentum. The dilated MPD was localized by palpation of the anterior surface and needle aspiration of clear pancreatic juice. The duct was completely laid open longitudinally from tail of the pancrease right up into neck and head (Fig. I). Towards the duodenal wall, the incision was kept within about a centimeter of the duodenum to avoid injury to the pancreatico duodenal vessels. Intraductal calculi were removed as far as possible from the entire length of the duct. Roux-en-Y jejunojenunostomy and side to side pancreatico-jejunostomy was done in two layers (Fig. II \& III). Operatively, all patients showed multiple stones in gray or olivary, polygon, round and antler shape. The large stone was 1.5 $\mathrm{cm}$ in diameter and most of them varied from $0.1 \mathrm{~cm}$ to 1.5 cm (Fig. IV). More than 10 stones were removed from one case and at least 3 stones removed from another case, average 3 to 10 stones removed. The key of this operation is to incise the pancreatic parenchyma along the line of the pancreatic duct to establish a big caliber of anastomosis after pancreatico-jejunostomy. It is good to drain along the line of the pancreatic duct effectively, thus making residual and recurrent stones drop in to the intestine through the anastomotic stoma, decompressing the pressure of the small pancreatic duct on the pancreatic parenchyma and postponing damage to gland cells and pancreatic enzyme secreting cells.

Postoperative follow up:

The main end point of the study was return to normal activities. The main outcome measures was pain relief, mortality and morbidity rates of the procedure. Postoperative evaluation was done, initially on a monthly basis for 3 months than 3 monthly for one year and yearly thereafter. Evaluation of the operative outcome in terms of pain relief after the operation, patients who were completely relieved of pain were grouped as satisfactory, patients still suffering from episodic moderate to severe pain requiring analgesics or persistent severe pain requiring regular analgesics/ opoids or hospitalization were grouped as unsatisfactory.

\section{Results:}

None of the patients were jaundiced at the time of presentation. Blood biochemistry shows mildly elevated serum amylase in 8 patients and hypoalbuminemia in 5 patients. Blood sugar was raised in 3 patients and

Table-I

Preoperative demographic clinical and investigational details of patients with pancreatic calculi:

\begin{tabular}{|c|c|}
\hline Age & Median 10 (range $7-15$ years) \\
\hline Sex & $10 \mathrm{M}, 4 \mathrm{~F}$ \\
\hline Mean duration of illness & 2.35 (range $1.5-4.5$ ) years \\
\hline \multirow[t]{2}{*}{ Positive findings on investigation } & Plain x-ray abdomen: 8 out of 10 (80\%)Ultrasonography: 14 out of $14(100 \%)$ \\
\hline & MRCP: 10 out of $10(100 \%) C T$ scan of abdomen: 4 out of $4(100 \%)$ \\
\hline Etiology & $\begin{array}{l}\mathrm{n}=14 \\
\text { Idiopathic }-12 \\
\text { Tropical pancreatitis }-2\end{array}$ \\
\hline \multirow[t]{3}{*}{ Symptomatology } & Severe pain - $14(100 \%)$ \\
\hline & Steatorrhea -2 \\
\hline & Weight loss -10 \\
\hline \multirow[t]{3}{*}{ Blood chemistry } & Hypoalbuminemia - 5 \\
\hline & Hyperamylasemia - 8 \\
\hline & Raised blood sugar - 3 \\
\hline Median MPD diameter & $7(5-10) \mathrm{mm}$ \\
\hline Pancreatic stone & 14 \\
\hline Pseudocyst & 1 \\
\hline
\end{tabular}


Table-II

Comparative preoperative and postoperative data of patients with pancreatic calculi.

\begin{tabular}{lll}
\hline Variable & Pre-operative & Post-operative \\
High blood sugar & 3 & None \\
Pain & Severe pain -14 & Completely relived-12 (85.7\%) \\
& & Moderate pain $-2(14.3 \%)$ \\
Steatorrhoea & 2 & None \\
Normal activities & None & All, within mean period of 6.5 months \\
Results of operation & & Satisfactory $-12(85.7 \%)$ \\
& & Unsatisfactory $-2(14.3 \%)$ \\
\hline
\end{tabular}

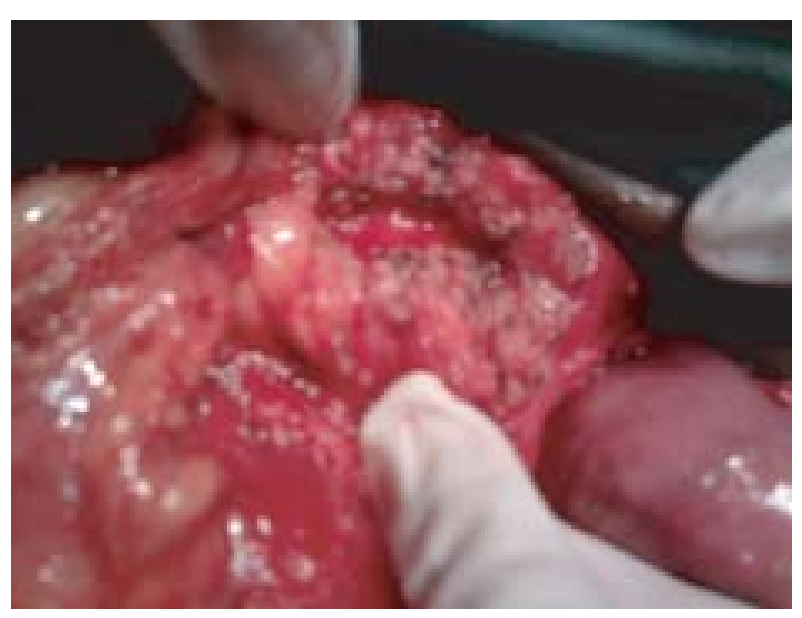

Fig.-1: Main pancreatic duct (laid open)

steatorrhoea in 2 patients. All biochemical parameter become normal post operatively. The mean operating time was 120 (range 90-150) min. No peroperative or post-operative death. No significant morbidity except wound infections in 2 patients. Median period of hospitalization was 8 (range 712) days, mean period of follow-up 22 (range 12-72) months.

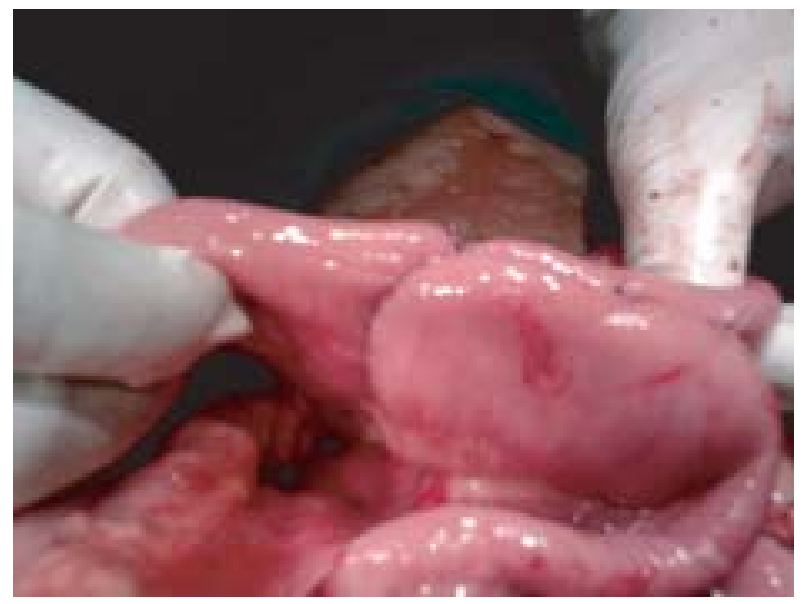

Fig.-2: Jejuno-jenunostomy
The operative results in terms of pain relief were satisfactory (no pain) in 12 (85.7\%) and unsatisfactory (moderate severe pain) in 2 (14.3\%) patients. High blood sugar of 3 patients and steatorrhoea of 2 patients become normal during the follow-up period. Two patients who had gross parenchymal calcification were older children continued to experience a moderate pain during followup. Comparative pre and post operative patient data are summarized in (Table-2).

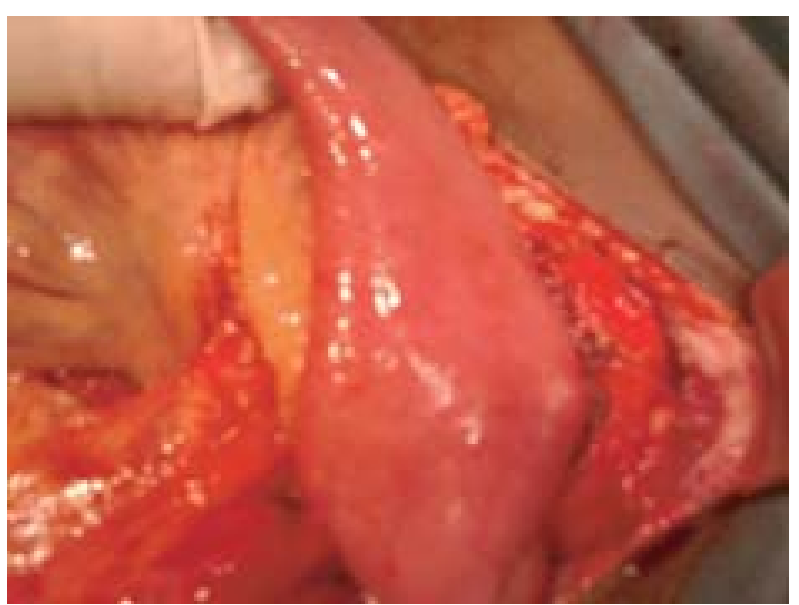

Fig.-3: Pancreatico jejunostomy (complete)

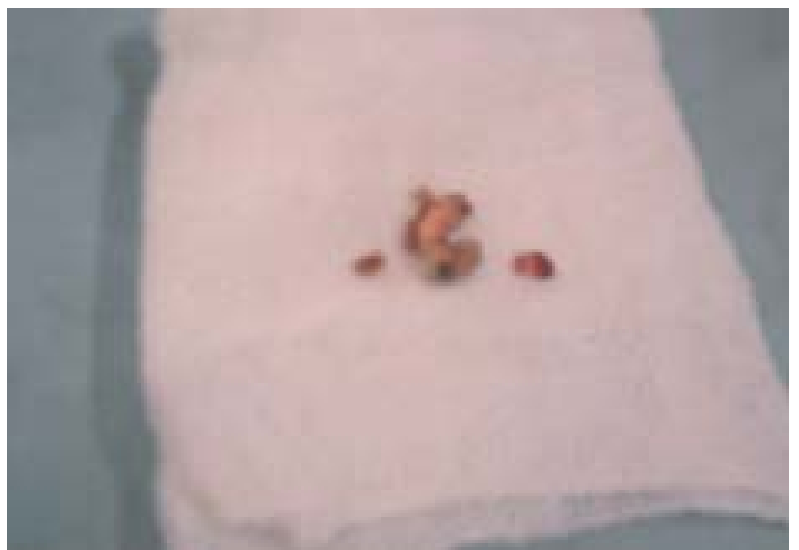

Fig.-4: Pancreatic duct calculi 


\section{Discussion:}

Because the pathogenesis of pancreatic stone is unknown, treatment of symptoms is predominantly adopted. The treatment principle is to remove all stones, relieve obstruction, ensure pancreatic fluid drainage, improve exocrine and endocrine function of the pancreas and relieve symptoms. Pain is the most common presentation while its intractability and interference with the lifestyle of the individual is the primary indication of surgical intervention ${ }^{3}$. The ideal operation for pancreatic duct stone should achieve pain relief without exacerbating exocrine and endocrine function while having low morbidity, mortality and easy to perform.

Longitudinal pancreatico-jejunostomy (LPJ) was found to be a safe procedure. The safety of the LPJ is evidenced by no significant morbidity and mortality. Many other authors have reported low mortality (range $0-4.4 \%)^{4}$. There was no death in our series in the per-operative or postoperative period and long-term follow-up period. Morbidity of this procedure is low. Post-operative wound infection developed in 2 (14.7\%) patients in this series and we managed it conservatively.

The key of this operation is to establish a big caliber of anastomosis after pancreatico-jejunostomy ${ }^{5}$. We had attempted to make the stoma as wide as possible and avoided to do it in patients with main pancreatic duct (MPD) $<5 \mathrm{~mm}$. It is good to drain along the line of the pancreatic duct effectively, thus making residual and recurrent stone to drop into the intestines through the anastomotic stoma, decompressing the pressure of the small pancreatic duct on the pancreatic parenchyma and postponing damage to gland cells and pancreatic secreting enzyme cells ${ }^{6}$. The definition of a successful outcome of surgery for pain relief is difficult. We equated it with satisfactory pain relief i.e. no pain. The satisfactory result in 12 (85.7\%) of the 14 patients followed up over a mean period of 22 months. Other authors have reported similar experiences $4,7,8$.

\section{Conclusion:}

Longitudinal pancreatico jejunostomy is a simple and safe procedure for ductal decompression which gives effective relief from intractable pain due to pancreatic duct calculi.

\section{References:}

1. Mariani A, Bernard JP, Provansal-Cheylan M. Difference of pancreatic stone morphology and content in patients with pancreatic lithiasis. Dig Dis Sci 1991;36:1509-1516.

2. Marrow CE, Cohen JI, Sutherland DR, Najarian JS. Chronic pancreatitis: long term surgical results of pancreatic duct drainage, pancreatic resection and near total pancreatectory and islet cell antofransplantation. Surgery 1984;96:608-15.

3. Devier J, Delhaye M, Gemer M. Pancreatic duct stones management. Gastro-intes Endoseclin N Am 1998; 8:163179.

4. Bradley EL. $3^{\text {rd }}$ Long term results of pancreatico-jejunostomy in patients with chronic pancreatitis. Am J Surg 1987; 153:207-13.

5. Wang YK, Mo YW, Liu YM, Wang JF. Clinical application of large calibre panceratic duct - Jejunostomy in treatment of pancreatic duct stone, Chin J Hepatobiliary Surg. 2001;7:564565 .

6. He ZP, Huang ZQ. Pancreatic stone and pancreatic cancer. Chin Dig J 1983; 3:40-41.

7. Pain JA, Knight MJ. Pancreaticogastrostomy: The preferred operation for pain relief in chronic pancratitis. Br J Surg 1988;75:220-2.

8. Chen J, Ling, Hu Y. The diagnosis and treatment of pancreatic duct stones. Chin J Hepatobiliary Surg, 2001; 7:564-565. 